Session 2265

\title{
WHAT ARE DIFFERENTIAL EQUATIONS: A Review of Curve Families
}

\author{
Andrew Grossfield \\ College of Aeronautics
}

\begin{abstract}
This paper is a review of curve families in light of their importance in a course on differential equations. Many texts depict curve families but do not treat them as an important mathematical concept that can greatly add to a student's comprehension of differential equations and continuous mathematics in general. It is not an accident that the solution of an nth order differential equation is an $n$ parameter curve family. It is not an accident that the solutions of linear differential equations are linear curve families. The forms, features and properties of curve families are discussed. Also to be discussed are the relations of one parameter curve families to surfaces in three dimensional space.
\end{abstract}

\section{What are Differential Equations?}

It is a good question. A student, desirous of answering the question, is not going to obtain a decent answer in most text books on Differential Equations. These texts assert "An equation with derivatives." Obviously! These equations have derivatives in them. But why? Who cares? Why would anyone be interested? That is as uninformative as telling students that functions are ordered pairs. What is needed is explanation.

Look at the simplest of the differential equations, those with just one derivative, the first order differential equations. Instead of focusing on the equation, focus on the solution, an equation with an independent variable, a dependent variable and a parameter. Solutions of these differential equations are equations in three variables.

A student who has completed a calculus course is familiar with equations in two variables which he/she may interpret as curves in a two dimensional plane. Now the student is embarking on a study of something completely new, a study of equations in three variables. Faculty, of course, are familiar with the graphical representations of equations in three variables as surfaces and curve families, but these ideas may not be familiar to students. Perhaps there may be a pedagogical advantage to ensuring that students understand the relationships between equations in three variables and surfaces and curve families. There may also be pedagogical advantages to providing at this time a discussion of surfaces and providing a discussion of curve families.

It is easy to show that beginning with the equation for curve family, $F\left(x, y, p i, p_{2}, p_{n}\right)=0$, in $\mathrm{n}$ parameters one can derive an nth order differential equation. However; it is only the simplest 
differential equations for which it is easy to derive the associated curve family. I am proposing that students be told that differential equations describe curve families and that solving a differential equation means finding the equation for the associated curve family. This would then naturally force a discussion of curve families: what they are and what there is to say about them. Most important, a curve family is not some graphical aid for solving a differential equation. It is the solution.

\section{Curve Families}

What are curve families? A set of curves that depend on a parameter. If the parameter is time, then the family could be said to be a moving curve. If the parameter, say $p$, is replaced with a function of the parameter, $f(p)$; then the curves are unchanged but the dependence on the parameter is altered. A curve family in one parameter, $p$, can be described by an implicit equation in three variables, $F(x, y, p)=0$. This same equation will describe a surface in three dimensional $\mathrm{x}-\mathrm{y}-\mathrm{p}$ space.

What is there to say about curve families? One could begin with a description of the forms, properties, and features of curve families. And then examine the relations of curve families to surfaces and also examine which characteristics of surfaces and curve families have meanings for the associated differential equation.

\section{Forms of Curve Families}

Below is a list of common forms for curve families:

- implicit,

- contour

- isolated dependent variable

- differential equation

- plane autonomous system

$$
\begin{aligned}
& \mathrm{H}(\mathrm{x}, \mathrm{y}, \mathrm{P})=0 \\
& \mathrm{P}=\mathrm{F}(\mathrm{x}, \mathrm{y}) \\
& \mathrm{Y}=\mathrm{G}(\mathrm{x}, \mathrm{P}) \\
& \mathrm{K}\left(\mathrm{y}^{\prime}, \mathrm{y}, \mathrm{x}\right)=0 \\
& \frac{d x}{d t}=\mathrm{F}(\mathrm{x}, \mathrm{y}) ; \quad \frac{d y}{d t}=\mathrm{G}(\mathrm{x}, \mathrm{y})
\end{aligned}
$$

The implicit form is ordinarily the common way to express a curve family. No variable is granted precedence over the others. If either variable, $\mathrm{p}$ or $\mathrm{y}$, can be isolated, the curve family can be described by either contour form or isolated independent variable form. The contour form provides an implicit relationship between the $\mathrm{x}$ and $\mathrm{y}$ variables for the individual curves of the family. This form is also of value for computing 3-dimensional plots of an associated surface. The isolated dependent variable form is good for computing the y values of the curves when the parameter values are known.

The differential equation relates the slope of a curve to each point through which the curve moves. Wondrously, this relationship can be enough to determine a whole family of curves that occupy the region under study. The study of the techniques for obtaining a form of the curve family without derivatives has constituted the traditional study of differential equations. The plane autonomous system comprises a popular form for studying non-linear dynamic systems, 
which is extendible to multi-variable and higher order systems. The variable $t$ is easily eliminated by division and the resulting differential equation is first order and linear in y' The phase plane plots that accompany the study of plane autonomous systems are no more than the solution curve families of the associated differential equation. Observe that the autonomous system can be linear while the associated differential equation is not.

\section{Properties of Curve Families}

Among interesting properties of curve families are

- normal (monotonic) curve families

- regular curve families

- linear curve families

Monotonic curve families are families whose curves move in one direction, as the parameter is incremented. A curve family is regular in a region if only one curve goes through each point and nearby curves are roughly parallel; that is, the curves appear as non-intersecting sfreamlines. These families are easy to visualize by drawing typical members. Linear curve families are families whose parametric forms are linear in the parameters. The solutions of linear differential equations are linear curve families.

\section{Features of Curve Families}

The simple curve families studied in an introductory DE course are regular mostly everywhere. The most distinguishing features are the points and lines where the regularity disappears. Isolated points, which lack regularity, called the critical points, are depicted in most introductory texts. Early in the course students should be provided with examples of the different types of critical points, -- the center, the saddle, the multiple-saddle, the proper node and the improper node. Families of level curves for a surface have centers at the maxima and minima of the surface. These families have saddle points where the surface appears as a saddle. In topographical maps saddle points represent the mountain passes. While commonly texts do provide the pictures of the critical points, the pictures are shown deep in the book in a chapter on systems rather than at the beginning in a section on curve families. (See Borelli $\sim 425$. See also Borelli, the intriguing table 11.1, pages $\mathbf{4 7 6}$ and 477, which illustrates how nonilinearities can affect the behavior at critical points of solutions of ODE's,)

Special lines associated with curve families are:

- separatrices or dividing lines

- envelopes

- boundaries

- limit cycles

These features also could be introduced graphically, early in the course as part of a discussion of curve families. 


\section{Connections between surfaces and curve families}

Comparing surfaces and curve families the following points should be made evident.

- The family of gradient curves of a surface is orthogonal to the family of level curves

- The family of level curves of a surface has a center at points corresponding to maxima or minima.

- The family of level curves of a surface has saddle points at points corresponding to passes.

- The family of gradient curves of a surface has proper nodes at points corresponding to maxima or minima.

\section{What about initial conditions?}

Conceptually differential equations do not come with initial conditions. They do describe curve families. The selection of a particular curve from a curve family has nothing to do with solving a differential equation. Many texts state that the solution of a differential equation is a function or curve. Such a definition requires that some kind of condition be attached to the differential equation in order to determine that unique solution. Historically the major numerical approaches to finding a solution function entailed constructing the curve incrementally starting with the initial conditions. But not all problems are initial condition problems. And sometimes it is the family that is desired. Let's avoid contusion. Tell your students that the solutions of differential equations are curve families. How they obtain a particular curve will depend on the problem at hand and the tools available for confronting that problem.

\section{Why study ordinary differential equations?}

In many cases the fundamental laws governing continuous physical systems, (such as dynamics, electronics, electromagnetics, thermodynamics and population dynamics) are relations between the local (point) values (such as position, speed and acceleration) of the variables of the system. Application of these laws results in differential equations. Solution of the differential equations yields global information about the systems such as the curves along which particles may move or the evolution of the system variables.

\section{Examples}

If examples are wanted, the second degree algebraic surfaces serve as a source of algebraically manipulable problems which exhibit some special features such as maxima, minima, saddlepoints, etc. Another source of examples can be obtained by relating the parameters in a multi-parameter family. Selecting functions $h(t), k(t)$, and $r(t)$ for the three parameter family of all circles in the plane, $(\mathrm{x}-\mathrm{h})^{2}+(\mathrm{y}-\mathrm{k})^{2}=\mathrm{r}^{2}$, results in a family of moving circles. These families can exhibit a variety of envelopes and boundaries. 


\section{Summary}

The above has been an introductory survey of curve families as to their major properties and their relations to differential equations and surfaces. This paper continues my efforts to focus attention on the topic structure and treatment of major concepts in college mathematics. I suggest that the reader examine my previous papers on functions and on curve plotting listed in the references below.

\section{References:}

1. Grossfield, Andrew "On the Intrinsic Structure of Calculus" Proceedings of the 1995 ASEE Annual Conference, Session 1265 (3 1 l-3 15)

2. Grossfield, Andrew "On the Classification of Functions and Curve Plotting" Proceedings of the 1990 ASEE Annual Conference, Session 2665 (1782-1784)

3. Robert L. Borrelli and Courtney S. Coleman, (1987) "Differential Equations: A Modeling Approach," Prentice Hall Publ. Englewood Cliffs, New Jersey

\section{Biographical Information}

Throughout his career Dr. Grossfield, has combined an interest in engineering design and mathematics. He earned his BSEE at the City College of New York. During the early sixties, he obtained an M. S. degree in mathematics at night while working full time during the day, designing circuitry for aerospace/avionics companies. As a Graduate Associate, pursuing a doctoral degree at the University of Arizona, he found himself in the odd position of both teaching calculus courses and taking courses in applied mathematics. Being caught in the middle made him acutely aware of the differences in mathematics, as viewed by the mathematician, as needed and used by the engineer and as presented to the student. He is licensed in New York as a Professional Engineer and is a member of ASEE, IEEE, and SIAM. His e-mail address is ai207@freenet.buffalo.edu 